\title{
Gene-Environment Interaction Effects of Peer Deviance, Parental Knowledge and Stressful Life Events on Adolescent Alcohol Use
}

\author{
Megan E. Cooke, ${ }^{1}$ Jacquelyn L. Meyers, ${ }^{2}$ Antti Latvala, ${ }^{3}$ Tellervo Korhonen, ${ }^{3,4}$ Richard J. Rose, ${ }^{5}$ \\ Jaakko Kaprio, ${ }^{3,6,7}$ Jessica E. Salvatore, ${ }^{1,8, *}$ and Danielle M. Dick ${ }^{8,9, *}$ \\ ${ }^{1}$ Virginia Institute for Psychiatric and Behavioral Genetics, Virginia Commonwealth University, Richmond, VA, USA \\ ${ }^{2}$ Department of Epidemiology, Columbia University, New York, NY, USA \\ ${ }^{3}$ Department of Public Health, University of Helsinki, Helsinki, Finland \\ ${ }^{4}$ Institute of Public Health and Clinical Nutrition, University of Eastern Finland, Kuopio, Finland \\ ${ }^{5}$ Department of Psychological and Brain Sciences, Indiana University, Bloomington, IN, USA \\ ${ }^{6}$ Department of Health, National Institute for Health and Welfare, Helsinki, Finland \\ ${ }^{7}$ Institute for Molecular Medicine, University of Helsinki, Helsinki, Finland \\ ${ }^{8}$ Department of Psychology, Virginia Commonwealth University, Richmond, VA, USA \\ ${ }^{9}$ Department of African American Studies, Virginia Commonwealth University, Richmond, VA, USA
}

\begin{abstract}
The purpose of this study was to address two methodological issues that have called into question whether previously reported gene-environment interaction (GxE) effects for adolescent alcohol use are 'real'. These issues are (1) the potential correlation between the environmental moderator and the outcome across twins and (2) non-linear transformations of the behavioral outcome. Three environments that have been previously studied (peer deviance, parental knowledge, and potentially stressful life events) were examined here. For each moderator (peer deviance, parental knowledge, and potentially stressful life events), a series of models was fit to both a raw and transformed measure of monthly adolescent alcohol use in a sample that included 825 dizygotic (DZ) and 803 monozygotic (MZ) twin pairs. The results showed that the moderating effect of peer deviance was robust to transformation, and that although the significance of moderating effects of parental knowledge and potentially stressful life events were dependent on the scale of the adolescent alcohol use outcome, the overall results were consistent across transformation. In addition, the findings did not vary across statistical models. The consistency of the peer deviance results and the shift of the parental knowledge and potentially stressful life events results between trending and significant, shed some light on why previous findings for certain moderators have been inconsistent and emphasize the importance of considering both methodological issues and previous findings when conducting and interpreting GxE analyses.
\end{abstract}

Keywords: gene-environment interaction, GxE, alcohol, peer deviance, parental knowledge, stressful life events

The study of GxE has received increasing attention over the past decade, as there has been growing recognition that the importance of genetic influences on behavioral traits can vary considerably as a function of the environment (Dick \& Kendler, 2012). This has been particularly true in the alcohol field, where the development of problems is (in some sense) contingent upon a particular environmental exposure: access to alcohol. Beyond exposure to alcohol, previous research has implicated peer deviance, parental knowledge, and stressful life events as moderators of the latent or measured genetic influence on adolescent alcohol use (Harden et al., 2008; Hicks et al., 2009; Miles et al., 2005). However, numerous statistical advances in the study of GxE have called into question the robustness of previously reported GxE findings (Rathouz et al., 2008; van der Sluis et al.,

RECEIVED 15 April 2015; ACCEPTED 14 July 2015. First published online 20 August 2015.

ADDRESS FOR CORRESPONDENCE: Megan Cooke, Virginia Institute for Psychiatric and Behavioral Genetics, Virginia Commonwealth University, PO Box 980126, Richmond, VA 23298-0126. E-mail: cookem3@vcu.edu

${ }^{*}$ Denotes shared last authorship as both individuals jointly provided supervision of the first author. 
2012). In the present study, we systematically re-examined these three environmental factors (parental knowledge, peer deviance, and potentially stressful life events) using new methods that account for the potential correlation between twins' level of the environment, unlike the statistical models that have historically been used to investigate GxE. In addition, we assessed whether non-linear transformations of the behavioral outcome would change previously reported GxE results, since some types of interactions have been shown to be scale dependent (Lynch \& Walsh, 1998; Mather \& Jinks, 1982).

Peer deviance, parental knowledge/monitoring, and stressful life events are three of the candidate environments that have been examined by multiple groups as moderators in the area of adolescent externalizing behavior (e.g., alcohol and drug use and conduct problems). A number of these studies found evidence that these factors significantly moderate genetic influences on alcohol use. Dick et al. (2007a) found that peer alcohol use moderated the heritability of adolescent alcohol use. They demonstrated that when more peers are using alcohol, the genetic influence on the adolescent's alcohol use is greater. Similarly, Harden et al. (2008) found that the effect of a best friend's substance use is dependent on the target adolescent's genetic liability; those adolescents with greater genetic liabilities toward substance use were more strongly affected by their best friend's substance use. With regards to parental monitoring, Dick et al. (2007b), using the classic univariate moderation model, showed that genetic influences on adolescent substance use were greater at lower levels of parental monitoring. In addition, Miles et al. (2005) found a moderating effect of parental characteristics on adolescent female alcohol use. They showed that at higher levels of parental closeness the genetic influences on adolescent alcohol use are lower. In all of these instances, genetic influences on alcohol use increased in the context of environments characterized by high levels of social opportunity to use and/or lack of social control (e.g., higher peer deviance, lower parental monitoring; Dick \& Kendler, 2012; Shanahan \& Hofer, 2005). The literature on stressful life events is more nuanced. Hicks et al. (2009), using a bivariate model, demonstrated that stressful life events moderated the genetic effect on externalizing disorders (including alcohol dependence) in adolescents. Again, genetic influences were greater in the context of more stressful life events. However, Button et al. (2007) found a positive genetic correlation between negative life events and externalizing behavior, but no GxE interaction.

The majority of these previous GxE studies used the univariate and bivariate models to study moderation as delineated in Purcell (2002). The univariate model allows for both linear and non-linear moderation of either the mean or the variance (conditional on the moderator) of the behavioral outcome. The bivariate model explicitly models the covariance between the behavioral outcome and the moderator, and allows for moderation of both the common and unique variance components. In a more recent examination of these models, van der Sluis et al. (2012) demonstrated that under certain conditions, the bivariate model has an increased false negative rate (Type II error) and the univariate model can result in false positives (Type I error). To address this problem, van der Sluis et al. (2012) extended Purcell's univariate model so that the behavioral outcome of each twin is corrected for the potential correlation with their own and their co-twin's value of the environmental moderator. Simulation studies showed that this extended univariate model accurately detected moderation and is more powerful than the bivariate model. However, if the covariance between the moderator and the behavioral outcome is moderated, the extended univariate model does not specify the location of the moderation, and the bivariate model provides the correct test. Conversely, in instances where the bivariate model shows no moderation of the covariance between the behavioral outcome and the moderator, the extended univariate model is the more powerful and appropriate model to test for moderation.

In addition to model selection, it has long been known that the scale of the behavioral outcome can have an effect on the detection of GxE. Mather and Jinks (1982) demonstrated, using data from several published examples, instances of GxE that varied in their robustness to non-linear transformation of the behavioral outcome. GxE can be detected as a result of the change in variance as the mean increases (heteroscedasticity). Non-linear transformations of the behavioral outcome (e.g., square root, logarithmic) in these cases will reduce the variance and can eliminate the interaction effect. However, interactions that are not dependent on this increased variance should be robust to transformations of scale. Despite this known concern, many studies of GxE fail to address issues of scaling.

In the present study, we systematically reexamined GxE effects for three environmental factors - peer deviance, parental knowledge, and potentially stressful life events that have been widely studied in the adolescent alcohol use literature and have been shown to moderate latent or measured genetic influences on adolescent externalizing behavior. Peer deviance, parental knowledge, and stressful life events can differ between twins and therefore have the potential to correlate with the co-twin's trait, leading to the detection of false positive GxE interactions. Following the recommendations of van der Sluis et al. (2012), we examined GxE for these environmental factors and adolescent alcohol use in the bivariate and extended univariate models (when relevant). In addition, we ran a series of analyses examining whether our results were robust to non-linear transformations of the alcohol use outcome. 


\section{Methods}

\section{Sample}

The sample for the present study came from the Finnish population-based FinnTwin 12 cohort, which is a longitudinal twin study designed to examine genetic and environmental influences on precursors to health outcomes, especially alcohol use (Kaprio et al., 2002). FinnTwin12 includes twins identified through Finland's Population Registry Center from five consecutive birth cohorts (1983-1987) collected from 1994 to 1998 comprised of 11- to 12-year-old twins. An initial family questionnaire was sent to all eligible twin families late in the year before the twins turned 12 years old, and received an $87 \%$ response rate. Additional baseline questionnaires were mailed to those families who returned the family questionnaire. Zygosity was assessed at this point by a well-validated questionnaire completed by both twins (Kaprio et al., 2002). Further follow-up questionnaires were sent at ages 14-17.5 years old, with the addition of agespecific items on health behaviors such as alcohol use and abuse. Here, we focus on data from the age 14 assessment ( $88 \%$ response rate), which included questions about alcohol use, parental knowledge, peer deviance, and stressful life events. The sample used for these analyses contained $803 \mathrm{MZ}$ twin pairs (409 female pairs and 394 male pairs) and 825 same-sex DZ twin pairs (386 female-female pairs and 436 male-male pairs). Female and male twin pairs were collapsed across zygosity in modeling consistent with previously published papers, since these analyses were intended as a follow-up to that work.

\section{Measures}

The adolescents reported on their alcohol use frequency in the past month using the following categorical response options: never, less often than once a month, about 1-2 times a month, or once a week or more. We then transformed this categorical variable into a semi-continuous variable indexing the approximate number of days (out of 30) the adolescent drank in the past month. The answer choices were recoded as following: never $=0$, less often than once a month $=0.5$, about 1-2 times a month $=1.5$, and once a week or more $=4.3$. Therefore, $0,0.5,1.5$, and 4.3 equal the approximate number of days in a month (30 days) that the adolescent drank corresponding to the ordinal response options (Dick et al., 2001).

The age 14 parental knowledge measure was the sum score of four adolescent self-report items $(\alpha=0.78)$ on the degree to which their parents know about their daily plans, activities and whereabouts, how they spend their money, and where/who they are with when not at home (Chassin et al., 1993). Responses were made on a fourpoint scale ranging from almost always to rarely or never. Items were reverse scored and summed so higher scores indicate more parental knowledge. The age 14 peer deviance measure was the sum score of four adolescent self-report items regarding the number of friends/acquaintances who drink, smoke, use drugs, and get into trouble at school $(\alpha=$ 0.77). Responses were made on a four-point scale ranging from none to more than five, and were summed such that higher scores indicate higher peer deviance. For both peer deviance and parental knowledge, sum scores were created and adjusted for missingness. Prorated sum scores were calculated for individuals who responded to at least half of the items by scaling up their raw sum score based on the number of missing items.

The age 14 stressful life events measure was a sum score of all stressful life events endorsed by the adolescent. Stressful life events were assessed using 13 dichotomous questions, with response options of yes or no, regarding changes in the adolescent's life, such as people moving away, death of a relative/friend, having conflicts, serious illness/injury, or birth of a sibling in the past two years. 'Yes' responses were summed to create a total life events score.

\section{Bivariate and Univariate GxE Twin Models}

For each moderator, we ran a bivariate Cholesky model with moderation (shown in Figure 1). The bivariate Cholesky model extends the classic twin design and allows for partitioning both the variance of each phenotype and their covariance. The variance and the covariance are partitioned into three sources - additive genetic factors (A), shared environmental factors $(\mathrm{C})$, and unique environmental factors (E) - based on the relationship between the MZ and DZ twin correlations. Moderation can be assessed in the bivariate model on the $\mathrm{A}, \mathrm{C}$, and $\mathrm{E}$ influences unique to the behavioral outcome and those shared with the moderator. Along with the $a, c$, and e path estimates, $\beta$ parameters, indicating the moderating effect of the moderator variable on each path, are also estimated. If the $\beta$ parameter is significantly different from zero, this indicates the existence of moderation on that pathway and the value of $\beta$ indicates the magnitude of the moderation. Moderation effects were tested first on the shared paths to determine whether it was appropriate to use the extended univariate model. If the moderation on the shared paths was significant, then moderation effects were tested on the unique paths for $\mathrm{A}, \mathrm{C}$, and $\mathrm{E}$ consecutively using the bivariate moderation model.

If moderation on all cross paths could be dropped, an extended univariate model was then fit (Figure 2). It also includes $\beta$ moderation parameters on each of the $a, c$, and e paths and moderation is tested in the same way as on the unique $\mathrm{a}, \mathrm{c}$, and e paths in the bivariate moderation model described above. The difference from the original Purcell univariate model is that estimation of the behavioral outcome value for each twin is corrected for both the value of twin's moderator and their co-twin's moderator. To assess moderation, the $\beta$ parameter was dropped on a, $c$, and $\mathrm{e}$ paths consecutively. 


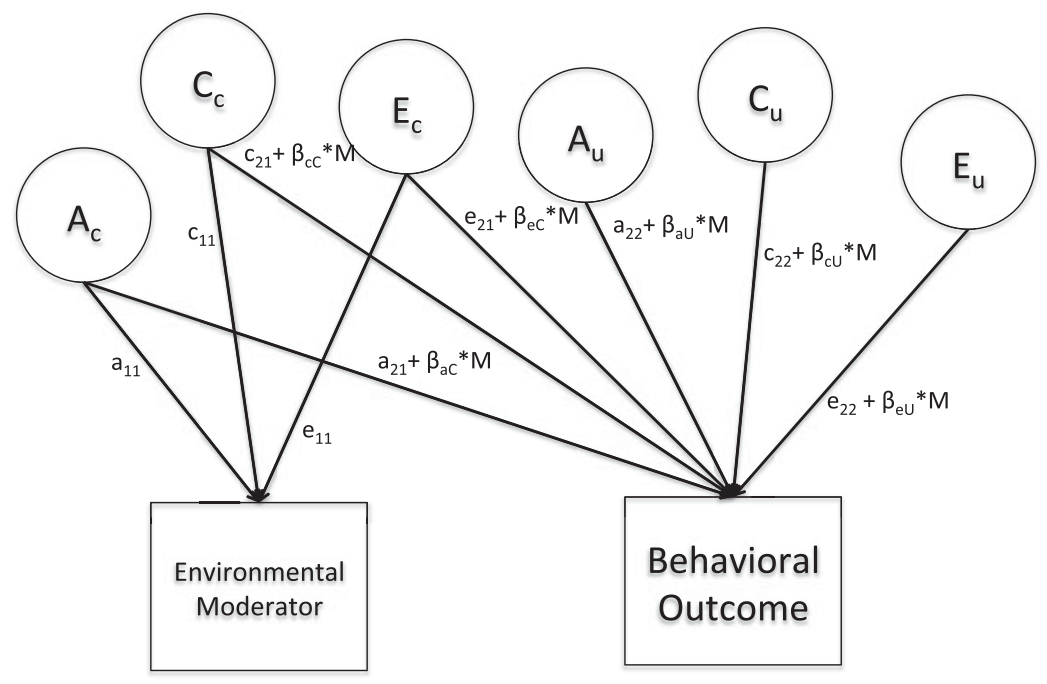

\section{FIGURE 1}

The bivariate moderation model. This figure illustrates the bivariate model (for one twin) with the addition of moderation components on the appropriate paths.

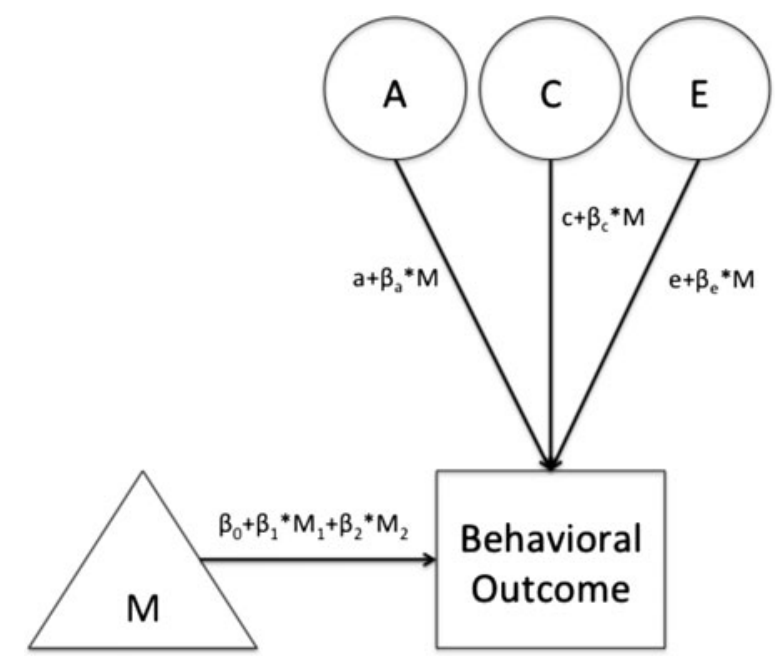

FIGURE 2

The extended univariate model. This figure illustrates the extended univariate moderation model (for one twin).

We ran a series of analyses: first, using the raw alcohol use frequency variable; second, using the square root transformed alcohol use frequency variable; and finally, using a logarithmic transformed alcohol use frequency variable. This was done to test how robust the findings were to different types of transformations in scale. Moderation can be falsely detected as an artifact of heteroscedasticity. A square root or logarithmic transformation in this case normalized the distribution by reducing the skew, testing if the effects were robust to changes in scale.
All analyses were run using Mx (Neale et al., 2003). Model selection was determined by the -2 log-likelihood difference method. For each parameter dropped, the $-2 \log$-likelihood of that model was compared to the -2 log-likelihood of the saturated model. If the submodel's -2 log-likelihood differed significantly according to a one degree of freedom chisquare test $(p<.05)$ from the saturated model, the model fit was judged to be significantly worse and the parameter should be retained in the model.

\section{Results}

Table 1 displays the descriptive statistics for all variables used in the subsequent analyses. The four variables were all modestly to moderately $(-0.09-0.44)$ correlated with each other in the expected directions. For example, higher levels of alcohol use were associated with higher levels of peer deviance, lower scores of parental knowledge and greater number of stressful life events.

Table 2 shows the twin correlations and the univariate twin model results for alcohol use frequency and the moderators. We found no mean differences in adolescent alcohol use between males and females. The variance in each of the environmental moderators and alcohol use frequency can be accounted for by genetic, shared environmental, and unique environmental influences. However, the size of the influence varies between the environmental moderators and alcohol use frequency.

\section{Peer Deviance}

We tested for moderation on the A, C, and E components that peer deviance and alcohol use frequency shared using the bivariate moderation model (shown in Table 3 ). 
TABLE 1

Descriptive Statistics

\begin{tabular}{llrllllll}
\hline & $N$ & Mean & SD & Min & Max & 1 & 2 & 3 \\
\hline 1. Peer deviance & 2,933 & 7.46 & 3.13 & 4 & 16 & & & \\
2. Parental knowledge & 2,930 & 13.45 & 2.14 & 4 & 16 & $-0.319^{*}$ & & \\
3. Stressful life events & 2,935 & 2.61 & 1.75 & 0 & 13 & $0.194^{*}$ & $-0.091^{*}$ & \\
4. Alcohol use & 2,918 & 0.39 & 0.82 & 0 & 4.3 & $0.438^{*}$ & $-0.307^{*}$ & $0.112^{*}$ \\
\hline Note: ${ }^{*} p<.01$. & & & & & & & &
\end{tabular}

\section{TABLE 2}

Twin Correlations and Univariate Results

\begin{tabular}{llllll}
\hline & MZ correlation & DZ correlation & $\mathrm{A}$ & $\mathrm{C}$ & $\mathrm{E}$ \\
\hline Peer deviance & $0.75^{*}$ & $0.68^{*}$ & 0.4 & 0.36 & 0.24 \\
& & & $(0.33-0.48)$ & $(0.29-0.42)$ & $(0.21-0.27)$ \\
Parental Knowledge & $0.58^{*}$ & $0.55^{*}$ & 0.35 & 0.26 & 0.40 \\
& & & $(0.23-0.46)$ & $(0.17-0.34)$ & $(0.36-0.44)$ \\
Stressful life events & $0.73^{*}$ & $0.60^{*}$ & 0.33 & 0.4 & 0.27 \\
& & & $(0.25-0.41)$ & $(0.33-0.47)$ & $(0.24-0.30)$ \\
Alcohol use & $0.71^{*}$ & $0.49^{*}$ & 0.53 & 0.17 & 0.30 \\
& & & $(0.43-0.63)$ & $(0.09-0.26)$ & $(0.27-0.33)$
\end{tabular}

Note: ${ }^{*} p<.01$; correlations are based on $803 \mathrm{MZ}$ pairs and $825 \mathrm{DZ}$ pairs. Confidence intervals are listed in parentheses under each ACE estimate.

TABLE 3

Peer Deviance Model Fit Statistics - Raw Variable

\begin{tabular}{lllllrrr}
\hline & $-2 \mathrm{LL}$ & $d f$ & \multicolumn{1}{l}{ AIC } & \multicolumn{1}{l}{ BIC } & \multicolumn{1}{c}{$\Delta$ LL } & $\Delta d f$ & $p$ \\
\hline Full model & $18,464.63$ & 5,752 & $6,960.63$ & $-11,695.06$ & & & \\
Drop $\beta_{\mathrm{aC}}$ & $18,465.04$ & 5,753 & $6,959.04$ & $-11,698.50$ & 0.40 & 1 & .53 \\
Drop $\beta_{\mathrm{cC}}$ & $18,476.15$ & 5,753 & $6,970.15$ & $-11,692.94$ & 11.51 & 1 & $<.01$ \\
Drop $\beta_{\mathrm{eC}}$ & $18,464.70$ & 5,753 & $6,958.70$ & $-11,698.66$ & 0.07 & 1 & .79 \\
Drop $\beta_{\mathrm{aC}}$ and $\beta_{\mathrm{eC}}$ & $18,462.46$ & 5,754 & $6,954.46$ & $-11,703.42$ & 2.17 & 2 & .99 \\
Drop $\beta_{\mathrm{aU}}$ & $18,524.29$ & 5,755 & $7,014.29$ & $-11,676.15$ & 61.83 & 1 & $<.01$ \\
Drop $\beta_{\mathrm{cU}}$ & $18,463.35$ & 5,755 & $6,953.35$ & $-11,706.61$ & 0.90 & 1 & .34 \\
Drop $\beta_{\mathrm{eU}}$ & $18,674.83$ & 5,755 & $7,164.83$ & $-11,600.88$ & 212.37 & 1 & $<.01$
\end{tabular}

Note: The full model refers to the full bivariate model seen in Figure 1. $-2 \mathrm{LL}=-2$ log-likelihood; $d f=$ degrees of freedom; AIC = Akaike Information Criterion, BIC = Bayesian Information Criterion; $\Delta \mathrm{LL}=$ difference in log-likelihood; $\Delta d f=$ difference in degrees of freedom.

The path estimates for the bivariate moderation model are shown in Figure 3. Since moderation was retained on the cross c path, it was not appropriate to use the extended univariate model. Therefore, we continued to test for moderation on the unique $a, c$, and e paths in the bivariate moderation model. As shown in Table 3, there were significant moderation effects on the unique $A$ and $E$ components. Figure 3 shows the changes in the raw variance of the shared and unique $\mathrm{A}, \mathrm{C}$, and $\mathrm{E}$ components as a function of peer deviance. Genetic and environmental influences increased under conditions of higher peer deviance, and were attenuated under conditions of lower peer deviance.

\section{Parental Knowledge}

Again using the bivariate moderation model, we tested for moderation on the $\mathrm{A}, \mathrm{C}$, and $\mathrm{E}$ components that parental knowledge and alcohol use share. As shown in Table 4, moderation was significant on the cross $c$ path. Therefore, it was not appropriate to use the extended univariate model. Moderation on the unique a, c, and e paths was then tested consecutively using the bivariate model. Table 4 shows that the moderating effects on the unique $\mathrm{A}$ and $\mathrm{E}$ components were retained. Figure 3 shows the changes in the raw variance of the shared and unique A, C, and E components as a function of parental knowledge. The genetic and environmental influences decreased under higher scores of parental knowledge and were greatest under lower parental knowledge.

\section{Stressful Life Events}

Following the same series of tests used for the other moderators, we first tested moderation on the cross paths using the bivariate moderation model. As shown in Table 5, moderation was retained on the cross $\mathrm{C}$ path, indicating it is inappropriate to use the extended univariate model to further test for moderation. Therefore, moderation was further tested on the unique a, c, and e paths using the bivariate model. Table 5 shows moderation could be dropped on the unique a path but not the unique $c$ and e paths. Figure 3 shows the changes in raw variance of the shared and unique $\mathrm{A}, \mathrm{C}$, and $\mathrm{E}$ as a function of stressful life events. The shared and unique environmental influences (but not 

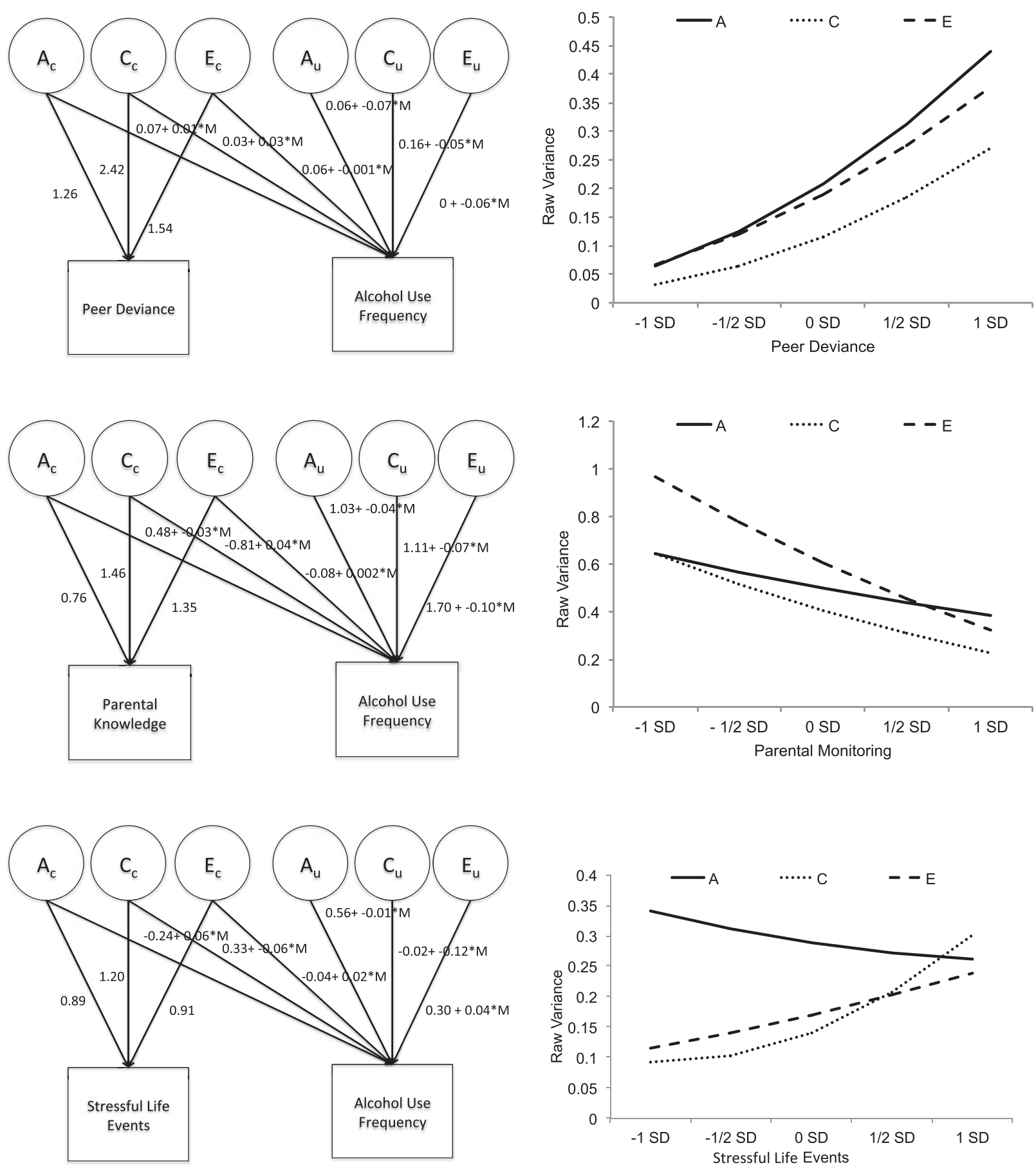

FIGURE 3

Bivariate moderation models and moderation results. On the left are the bivariate moderation models with the moderation paths for each of the moderators. On the right are graphs of the raw variance of $A, C$, and $E$ as a function of the moderators on both the unique and cross paths.

additive genetic influences) were greatest under conditions of greater potentially stressful life events and diminished at lower numbers of potentially stressful life events. The additive genetic influences did not change significantly as a function of the number of potentially stressful life events.

\section{Transformed Alcohol Frequency Analyses}

We reran our GxE models using a square root transformed and a logarithmic transformed alcohol use frequency variable in order to examine the robustness of the results that emerged using the raw alcohol variable. The square root 
TABLE 4

Parental Knowledge Model Fit Statistics — Raw Variable

\begin{tabular}{|c|c|c|c|c|c|c|c|}
\hline & $-2 \mathrm{LL}$ & $d f$ & $\mathrm{AlC}$ & $\mathrm{BIC}$ & $\Delta \mathrm{LL}$ & $\Delta d f$ & $p$ \\
\hline Full model & $17,227.25$ & 5,733 & $5,761.25$ & $-12,236.69$ & & & \\
\hline Drop $\beta_{\mathrm{aC}}$ & $17,227.90$ & 5,734 & $5,759.90$ & $-12,239.99$ & 0.66 & 1 & .42 \\
\hline Drop $\beta_{\mathrm{c} C}$ & $17,231.57$ & 5,734 & $5,763.57$ & $-12,238.16$ & 4.32 & 1 & .04 \\
\hline Drop $\beta_{\mathrm{e}}$ & $17,227.29$ & 5,734 & $5,759.29$ & $-12,240.30$ & 0.04 & 1 & .85 \\
\hline Drop $\beta_{\mathrm{ac}}$ and $\beta_{\mathrm{ec}}$ & $17,231.99$ & 5,735 & $5,761.99$ & $-12,241.59$ & 4.74 & 2 & .09 \\
\hline Drop $\beta_{\mathrm{aU}}$ & $17,239.15$ & 5,736 & $5,767.15$ & $-12,241.65$ & 7.16 & 1 & .01 \\
\hline Drop $\beta_{\mathrm{cU}}$ & $17,235.26$ & 5,736 & $5,763.26$ & $-12,243.59$ & 3.27 & 1 & .07 \\
\hline Drop $\beta_{\mathrm{eU}}$ & $17,519.59$ & 5,736 & $6,047.59$ & $-12,101.43$ & 287.60 & 1 & $<.01$ \\
\hline
\end{tabular}

\section{TABLE 5}

Stressful Life Events Bivariate Moderation Model — Raw Variable

\begin{tabular}{|c|c|c|c|c|c|c|c|}
\hline & $-2 \mathrm{LL}$ & $d f$ & $\mathrm{AlC}$ & $\mathrm{BIC}$ & $\Delta \mathrm{LL}$ & $\Delta d f$ & $p$ \\
\hline Full model & $16,742.96$ & 5,754 & $5,234.96$ & $-12,565.16$ & & & \\
\hline Drop $\beta_{\mathrm{ac}}$ & $16,745.56$ & 5,755 & $5,235.56$ & $-12,567.50$ & 2.60 & 1 & .11 \\
\hline Drop $\beta_{\mathrm{cC}}$ & $16,747.31$ & 5,755 & $5,237.31$ & $-12,566.63$ & 4.35 & 1 & .04 \\
\hline Drop $\beta_{\mathrm{e}} \mathrm{C}$ & $16,745.76$ & 5,755 & $5,235.76$ & $-12,567.40$ & 2.80 & 1 & .09 \\
\hline Drop $\beta_{\mathrm{ac}}$ and $\beta_{\mathrm{ec}}$ & $16,753.55$ & 5,756 & $5,241.55$ & $-12,567.15$ & 10.59 & 2 & .01 \\
\hline Drop $\beta_{\mathrm{aU}}$ & $16,756.79$ & 5,757 & $5,242.79$ & $-12,569.16$ & 3.24 & 1 & .07 \\
\hline Drop $\beta_{\mathrm{cU}}$ & $16,774.96$ & 5,757 & $5,260.96$ & $-12,560.08$ & 21.41 & 1 & $<.01$ \\
\hline Drop $\beta_{\mathrm{eU}}$ & $16,793.17$ & 5,757 & $5,279.17$ & $-12,550.97$ & 39.63 & 1 & $<.01$ \\
\hline
\end{tabular}

Note: The full model refers to the full bivariate model seen in Figure 1. $-2 \mathrm{LL}=-2$ log-likelihood; $d f=$ degrees of freedom; AIC = Akaike Information Criterion, BIC = Bayesian Information Criterion; $\Delta \mathrm{LL}=$ difference in log-likelihood; $\Delta \mathrm{df}=$ difference in degrees of freedom.

\section{TABLE 6}

Peer Deviance Model Fit Statistics — Transformed Variable

\begin{tabular}{lllllllr}
\hline & $-2 \mathrm{LL}$ & $d f$ & AIC & BIC & $\Delta \mathrm{LL}$ & $\Delta d f$ & $\mathrm{p}$ \\
\hline Full model & $16,315.54$ & 5,752 & $4,811.54$ & $-12,769.61$ & & & \\
Drop $\beta_{\mathrm{aC}}$ & $16,355.19$ & 5,753 & $4,849.19$ & $-12,753.42$ & 39.65 & 1 & $<.01$ \\
Drop $\beta_{\mathrm{cC}}$ & $16,380.22$ & 5,753 & $4,874.22$ & $-12,740.91$ & 64.68 & 1 & $<.01$ \\
Drop $\beta_{\mathrm{eC}}$ & $16,354.32$ & 5,753 & $4,848.32$ & $-12,753.85$ & 38.79 & 1 & $<.01$ \\
Drop $\beta_{\mathrm{aU}}$ & $16,321.96$ & 5,753 & $4,815.96$ & $-12,770.03$ & 6.43 & 1 & .01 \\
Drop $\beta_{\mathrm{cU}}$ & $16,324.20$ & 5,753 & $4,818.20$ & $-12,768.92$ & 8.66 & 1 & $<.01$ \\
Drop $\beta_{\mathrm{eU}}$ & $16,386.60$ & 5,753 & $4,880.60$ & $-12,737.71$ & 71.06 & 1 & $<.01$ \\
\hline Note: The full model refers to the full bivariate model seen in Figure 1. $-2 \mathrm{LL}=-2$ log- \\
likelihood; $d f=$ degrees of freedom; AIC = Akaike Information Criterion, BIC = \\
Bayesian Information Criterion; $\Delta \mathrm{LL}=$ difference in log-likelihood; $\Delta d f=$ difference \\
in degrees of freedom.
\end{tabular}

transformed variable and the logarithmic transformed variable were highly correlated, $r(4,707)=0.99, p<.01$. The results were consistent between the two transformations and only the results from the square root transformed variable are presented here.

Peer deviance. Similar to the analyses using the raw variable, we tested for moderation on all cross paths between peer deviance and alcohol use frequency and on all paths unique to alcohol use frequency using the bivariate moderation model. As shown in Table 6, evidence for moderation was found on cross and unique a, c, and e paths. Genetic and environmental influences were greater at higher levels of peer deviance and reduced at low levels of peer deviance (see Supplemental Figure).
Parental knowledge. As shown in Table 7a, moderation on the cross A, C, and E paths could be dropped; therefore, the extended univariate model was used to further test for moderation. Moderation could only be retained on the E path in the extended univariate model, shown in Table 7b. Therefore, genetic and shared environmental influences did not change significantly across levels of parental knowledge. Unique environmental influences were greater at lower scores on the parental knowledge variable (riskier environment) and reduced at higher scores on the parental knowledge variable (see Supplemental Figure 1).

Stressful life events. As shown in Table $8 \mathrm{a}$, moderation could be dropped on all three of the cross paths. Therefore, the extended univariate model was used to further test moderation. Table $8 \mathrm{~b}$ shows moderation could be retained on 
TABLE 7a

Parental Knowledge Bivariate Model Fit Statistics - Transformed Variable

\begin{tabular}{llllllll}
\hline & $-2 \mathrm{LL}$ & $d f$ & $\mathrm{AIC}$ & $\mathrm{BIC}$ & $\Delta \mathrm{LL}$ & $\Delta d f$ & $\mathrm{p}$ \\
\hline Full Model & $15,016.96$ & 5,733 & $3,550.96$ & $-13,341.83$ & & & \\
Drop $\beta_{\mathrm{aC}}$ & $15,018.95$ & 5,734 & $3,550.95$ & $-13,344.47$ & 1.99 & 1 & .16 \\
Drop $\beta_{\mathrm{cC}}$ & $15,020.80$ & 5,734 & $3,552.80$ & $-13,343.55$ & 3.84 & 1 & .05 \\
Drop $\beta_{\mathrm{eC}}$ & $15,017.26$ & 5,734 & $3,549.26$ & $-13,345.32$ & 0.30 & 1 & .58 \\
Drop $\beta_{\mathrm{aC}} \& \beta_{\mathrm{eC}}$ & $15,018.95$ & 5,735 & $3,548.95$ & $-13,348.11$ & 1.99 & 2 & .37 \\
Drop $\beta_{\mathrm{aU}}$ & $15,021.97$ & 5,736 & $3,549.97$ & $-13,350.23$ & 3.02 & 1 & .08 \\
Drop $\beta_{\mathrm{cU}}$ & $15,023.36$ & 5,736 & $3,551.36$ & $-13,349.54$ & 4.41 & 1 & .04 \\
Drop $\beta_{\mathrm{eU}}$ & $15,128.97$ & 5,736 & $3,656.97$ & $-13,296.73$ & 110.03 & 1 & $<.01$ \\
\hline
\end{tabular}

Note: The full model refers to the full bivariate model seen in Figure 1. $-2 \mathrm{LL}=-2$ log-likelihood; $d f=$ degrees of freedom; $\mathrm{AIC}=$ Akaike Information Criterion, $\mathrm{BIC}=$ Bayesian Information Criterion; $\Delta \mathrm{LL}=$ difference in log-likelihood; $\Delta d f=$ difference in degrees of freedom.

TABLE 7b

Parental Knowledge Extended Univariate Model Fit Statistics - Transformed Variable

\begin{tabular}{lllllllr}
\hline & -2LL & $d f$ & AIC & BIC & $\Delta \mathrm{LL}$ & $\Delta d f$ & $p$ \\
\hline Full model & $3,048.12$ & 2,854 & $-2,659.88$ & $-8,855.64$ & & & \\
Drop $\beta_{\mathrm{a}}$ & $3,051.54$ & 2,855 & $-2,658.46$ & $-8,857.56$ & 3.42 & 1 & .06 \\
Drop $\beta_{\mathrm{c}}$ & $3,052.09$ & 2,855 & $-2,657.91$ & $-8,857.29$ & 3.97 & 1 & .05 \\
Drop $\beta_{\mathrm{e}}$ & $3,157.43$ & 2,855 & $-2,552.57$ & $-8,804.62$ & 109.31 & 1 & $<.01$ \\
\hline Note: The full model refers to the full extended univariate model seen in Figure 2. $-2 \mathrm{LL}=-2$ \\
log-likelihood; df = degrees of freedom; AIC = Akaike Information Criterion, BIC = \\
Bayesian Information Criterion; $\Delta \mathrm{LL}=$ difference in log-likelihood; $\Delta$ df = difference in \\
degrees of freedom.
\end{tabular}

TABLE 8a

Stressful Life Events Bivariate Model Fit Statistics — Transformed Variable

\begin{tabular}{lllllrlr}
\hline & $-2 \mathrm{LL}$ & $d f$ & AIC & BIC & $\Delta$ LL & $\Delta d f$ & $p$ \\
\hline Full model & $14,112.29$ & 5,754 & $2,604.29$ & $-13,880.50$ & & & \\
Drop $\beta_{\mathrm{aC}}$ & $14,113.75$ & 5,755 & $2,603.75$ & $-13,883.41$ & 1.46 & 1 & .23 \\
Drop $\beta_{\mathrm{cC}}$ & $14,115.15$ & 5,755 & $2,605.15$ & $-13,882.71$ & 2.86 & 1 & .09 \\
Drop $\beta_{\mathrm{eC}}$ & $14,115.47$ & 5,755 & $2,605.47$ & $-13,882.55$ & 3.17 & 1 & .08 \\
Drop $\beta_{\mathrm{aC}}, \beta_{\mathrm{cC}}$ and $\beta_{\mathrm{eC}}$ & $14,122.18$ & 5,757 & $2,608.18$ & $-13,886.47$ & 9.89 & 3 & .02 \\
Drop $\beta_{\mathrm{aU}}$ & $14,127.36$ & 5,758 & $2,611.36$ & $-13,887.52$ & 5.18 & 1 & .02 \\
Drop $\beta_{\mathrm{cU}}$ & $14,133.61$ & 5,758 & $2,617.61$ & $-13,884.39$ & 11.43 & 1 & .01 \\
Drop $\beta_{\mathrm{eU}}$ & $14,125.34$ & 5,758 & $2,609.34$ & $-13,888.53$ & 3.16 & 1 & .08 \\
\hline
\end{tabular}

Note: The full model refers to the full bivariate model seen in Figure 1. $-2 \mathrm{LL}=-2$ log-likelihood; $d f=$ degrees of freedom; AIC = Akaike Information Criterion, BIC = Bayesian Information Criterion; $\Delta \mathrm{LL}=$ difference in log-likelihood; $\Delta d f=$ difference in degrees of freedom.

TABLE 8b

Stressful Life Events Extended Univariate Model Fit Statistics - Transformed Variable

\begin{tabular}{lllllrlr}
\hline & -2LL & df & \multicolumn{1}{l}{ AIC } & \multicolumn{1}{l}{ BIC } & $\Delta$ LL & $\Delta d f$ & $p$ \\
\hline Full model & $3,561.66$ & 2,865 & $-2,168.34$ & $-8,643.83$ & & & \\
Drop $\beta_{\mathrm{a}}$ & $3,566.79$ & 2,866 & $-2,165.21$ & $-8,644.90$ & 5.14 & 1 & .02 \\
Drop $\beta_{\mathrm{c}}$ & $3,572.70$ & 2,866 & $-2,159.30$ & $-8,641.95$ & 11.04 & 1 & $<.01$ \\
Drop $\beta_{\mathrm{e}}$ & $3,564.92$ & 2,866 & $-2,167.08$ & $-8,645.84$ & 3.26 & 1 & .07 \\
\hline
\end{tabular}

Note: The full model refers to the full extended univariate model seen in Figure 2. $-2 \mathrm{LL}=-2$ log-likelihood; $d f=$ degrees of freedom; $\mathrm{AIC}=$ Akaike Information Criterion, $\mathrm{BIC}=$ Bayesian Information Criterion; $\Delta \mathrm{LL}=$ difference in log-likelihood; $\Delta d f=$ difference in degrees of freedom.

the a and c paths and dropped on the e path. At high levels of stressful life events, the shared environmental influences were greatest, whereas at low levels of stressful life events the shared environmental influences were attenuated. The reverse is true for the genetic influences: genetic influences were greatest at low levels of stressful life events and smallest at high levels of stressful life events (see Supplemental Figure). 


\section{Discussion}

There has been a history of skepticism surrounding GxE interactions (Duncan \& Keller, 2011; Eaves, 2006; Kendler \& Gardner, 2010; McClelland \& Judd, 1993). Such skepticism reiterates the importance of approaching $\mathrm{GxE}$ analyses cautiously and systematically, and the need to revisit previous findings when new statistical models become available in the field. The results of the current study, where we examined GxE effects for adolescent alcohol use frequency in the context of three salient environments (parental knowledge, peer deviance, and potentially stressful life events), address two issues that are relevant for assessing the robustness of previous GxE findings in the area of adolescent externalizing behavior: first, the importance of evaluating different models to test for $\mathrm{GxE}$; and second, the importance of assessing whether any effects that emerge are robust to non-linear transformations of the behavioral outcome. We discuss each of these points in turn.

The moderating effect of peer deviance on adolescent alcohol use was robust to both of these tests. After systematically choosing the best fitting model, peer deviance was shown to moderate the additive genetic, shared environmental, and unique environmental influences on alcohol use. These results were found for both the raw and transformed versions of the alcohol use variable. The consistency of these findings across transformation is further supported by the literature. Using the same Finnish sample Dick et al. (2007a) found that friends' alcohol use significantly moderated the additive genetic, shared environmental, and unique environmental influences on adolescents' alcohol use at age 17.5. In a different sample, Harden et al. (2008) found that the effect of a best friend's substance use was greater among adolescents with higher genetic liabilities. Peer deviance is also found to interact with genetic risk for both alcohol use disorder specifically and externalizing phenotypes in general on level of alcohol consumption (Kendler et al., 2011). In all the aforementioned studies, at higher levels of peer deviance/substance use there were greater genetic effects. The consistent support in the literature and the stability of the GxE effects across different models and transformations further emphasizes the moderating effects of peer deviance on the genetic influences on adolescent alcohol use as a robust effect.

The effects associated with parental knowledge analyses showed greater dependency on the scaling of adolescent alcohol use. In the GxE models using the raw alcohol use frequency variable, we observed that parental knowledge moderated the additive genetic, shared environmental and unique environmental influences. However, when using a non-linear square root transformed alcohol use variable, the moderating effects of genetic influences and shared environmental influences could be dropped in the model fitting procedure, though they still showed a trend $(p=.06$, $p=.05)$ in the expected direction, whereby genetic and shared environmental influences increased under conditions of lower parental knowledge. While there is still a trend in the expected direction, the fact that the moderating effects in this case are affected by the distribution of the behavioral outcome may call into question the stability of the original findings in the raw variable (Lynch \& Walsh, 1998; Mather \& Jinks, 1982). Previously, using the same Finnish twin sample, Dick et al. (2007a) reported moderating effects of parental monitoring on adolescent smoking but not drinking. Our analyses differed from those of Dick et al. (2007a) in that we used a semi-continuous measure of alcohol use frequency and assessed these findings using the bivariate moderation model and the newer extended univariate model. These differences potentially explain our findings of significant moderation of genetic influences with the raw variable but only a trend in the transformed variable.

The decreased statistical significance across transformations does not rule out the possibility that parental knowledge is an important environmental moderator of genetic predispositions on adolescent substance use. Studies have shown an interaction between parenting characteristics and specific genetic polymorphisms on general substance use and externalizing behavior (Brody et al., 2009; Dick et al., 2009). Additionally, parental knowledge may not be the most important parental characteristic for alcohol use specifically. Miles et al. (2005) found that parental closeness was the most important parental characteristic for moderating the genetic influences on adolescent alcohol use. In the context of these previous findings, the change in our findings from statistically significant to trend-level across transformation, it is reasonable to conclude that the relationship between parental monitoring and adolescent alcohol use is subtler than that between peer deviance and adolescent alcohol use. Alcohol use, and specifically problematic use, can be assessed and represented in a variety of ways. Because there is no true metric for alcohol problems, inconsistent findings with one scaling versus another only serve to remind us that we are testing for statistical interactions rather than biological interactions (Kendler \& Gardner, 2010) and that conclusions about these interactions cannot be made absent of underlying theory.

Of the moderators tested, the relationship described in the literature between alcohol use and stressful life events was the most tenuous (Veenstra et al., 2006). Using the raw alcohol use variable, we found (in the bivariate model) that potentially stressful life events only significantly moderated the environmental influences on the raw alcohol use variable, with the moderating effect on genetic influences yielding a trending effect $(p=.07)$. Using the transformed variable, we found (in the both the bivariate and the extended univariate models) that potentially stressful life events significantly moderated the genetic and shared environmental influences on the transformed variable. Similar to our findings with the raw variable, Button et al. (2008) found 
that negative life events moderated the influence of unique environmental factors but not additive genetic or shared environmental factors on externalizing behavior. However, Hicks et al. (2009) found that stressful life events moderated additive genetic, shared environmental, and unique environmental influences on externalizing behavior (including alcohol use), which is similar to our findings with the transformed variable. Although both the raw and transformed results are consistent with previous findings, the change from trend to statistical significance of these results across transformations within the Finnish sample provides a useful example of the potential effect of heteroscedasticity on GxE effects. Heteroscedasticity can produce biased standard errors. Transforming the alcohol use variable reduces the heteroscedasticity leading to unbiased standard errors and a significant GxE finding in the transformed (but not raw) alcohol use variable. In addition, although similar events may have been assessed in each of these studies, the extent to which they were perceived to be stressful was not included in these analyses (i.e., the events measured in this study are potentially stressful life events). These factors may partially explain some of the ambiguity in the nature of the GxE relationship for adolescent alcohol use and potentially stressful life events.

Considering the raw and transformed results across the three moderators as a set, the question of why some moderators are more susceptible to transformation than others still remains. We tested if there were a differential number of bivariate outliers across each moderator and the alcohol use variable and found the number of bivariate outliers to be consistent across the moderators with both the raw and transformed alcohol use variable. Therefore, the difference in susceptibility to transformation between environmental moderators is unlikely due to the bivariate distributions of these moderators. Instead, this difference could be in part due to the lack of an absolute metric for the environment. Latent GxE effects capture changes in heritability across different levels of the environment. And, like all heritability estimates, these estimates are sample specific (Verhulst et al., 2015). Therefore, GxE findings have the potential to vary both across difference studies and when the distribution of the outcome variable changes as the result of a non-linear transformation. An additional related complication is that, just like the complex traits of interest in GxE studies (including alcohol use related behaviors), these measured environments have no 'true metric' (Dick et al., 2015). Therefore, each measurement of an environment may (or may not) tap into one (or multiple) aspects of the environment that are relevant to the development of the complex trait.

The findings from the present study should be interpreted in the context of several limitations. First, we were unable to formally test whether the moderating effects of peer deviance, parental monitoring, or potentially stressful life events on adolescent alcohol use differed by sex. The basic GxE models described in this article require relatively large sample sizes to detect effects. Therefore, with the increased complexity of testing sex differences in moderation models we would not have had sufficient power. Further studies are needed to assess the potential important differences in how these moderators affect male and female alcohol use during adolescence. Second, family and twin data-based models are not informative as to which genes are responsible for the interactions discovered in twin models. Accordingly, studies of latent GxE can tell us about environmental factors that moderate overall genetic influence, but not specific genes or facets of a predisposition that may interact with environmental factors.

In conclusion, we tested the robustness of three moderators previously associated with adolescent alcohol use. We assessed these effects using a systematic pipeline of $\mathrm{GxE}$ models, comprised of the bivariate twin model and the extended univariate model, to examine moderators that could potentially vary between twins. In addition, we tested if the effects were robust following two non-linear transformations of the alcohol use measure. We found that when the assumptions of the extended univariate model are met, the results generated do not differ from those of the corresponding bivariate moderation model. With regards to parental knowledge, the moderating effect showed the same pattern across the raw and transformed outcome variables. The effect was borderline significant after transformation, indicating a more subtle relationship between parental monitoring and adolescent alcohol use. The moderating effect of potentially stressful life events on the genetic influences on alcohol use showed a trend in the raw variable and was significant using a transformed variable. Finally, along with having the strongest literature support, the effects of peer deviance were robust to both the model selection and transformation. The three distinct results from these three moderators emphasize the need for replication of $\mathrm{GxE}$ findings in the context of new models that address previous statistical limitations, and testing the robustness of GxE effects following a non-linear transformation of the dependent variable.

\section{Acknowledgments}

The Finnish Twin studies have been supported by the National Institute of Alcohol Abuse and Alcoholism (grants AA12502, AA00145, and AA-09203 to RJR and AA015416A1 and K02AA018755 to DMD), the Academy of Finland (grants 100499, 205585, 141054 and 118555, 265240, 263278, and 264146 to JK); and the Academy of Finland Centre of Excellence Programme (grants 213506, 129680 to JK). JES was supported by T32MH20030-14 and F32AA022269. MEC was supported by UL1TR000058 and R25DA026119-06A1. The contents of the paper are solely the responsibility of the authors and do not necessarily represent the official views of the funders. 


\section{Supplementary Material}

To view supplementary material for this article, please visit http://dx.doi.org/10.1017/thg.2015.56.

\section{References}

Brody, G. H., Beach, S. R., Philibert, R. A., Chen, Y. F., Lei, M. K., Murry, V. M., \& Brown, A.C. (2009). Parenting moderates a genetic vulnerability factor in longitudinal increases in youths' substance use. Journal of Consulting \& Clinical Psychology, 77, 1-11.

Button, T. M., Corley, R. P., Rhee, S. H., Hewitt, J. K., Young, S. E., \& Stallings, M. C. (2007). Delinquent peer affiliation and conduct problems: A twin study. Journal of Abnormal Psychology, 116, 554-564.

Button, T. M., Lau, J. Y., Maughan, B., \& Eley, T. C. (2008). Parental punitive discipline, negative life events and geneenvironment interplay in the development of externalizing behavior. Psychological Medicine, 38, 29-39.

Chassin, L., Pillow, D. R., Curran, P. J., Molina, B. S., \& Barrera, M., Jr. (1993). Relation of parental alcoholism to early adolescent substance use: A test of three mediating mechanisms. Journal of Abnormal Psychology, 102, 3-19.

Dick, D. M., Agrawal, A., Keller, M. C., Adkins, A., Aliev, F., Monroe, S., ... Sher, K. J. (2015). Candidate geneenvironment interaction research. Perspectives on Psychological Science, 10, 37-59.

Dick, D. M., \& Kendler, K. S. (2012). The impact of geneenvironment interaction on alcohol use disorders. Alcohol Research, 34, 318-324.

Dick, D. M., Latendresse, S. J., Lansford, J. E., Budde, J. P., Goate, A., Dodge, K. A., .. Bates, J. E. (2009). Role of GABRA2 in trajectories of externalizing behavior across development and evidence of moderation by parental monitoring. Archives of General Psychiatry, 66, 649-657.

Dick, D. M., Pagan, J. L., Viken, R., Purcell, S., Kaprio, J., Pulkkinen, L., \& Rose, R. J. (2007a). Changing environmental influences on substance use across development. Twin Research and Human Genetics, 10, 315-326.

Dick, D. M., Rose, R. J., Viken, R. J., Kaprio, J., \& Koskenvuo, M. (2001). Exploring gene-environment interactions: Socioregional moderation of alcohol use. Journal of Abnormal Psychology, 110, 625-632.

Dick, D. M., Viken, R., Purcell, S., Kaprio, J., Pulkkinen, L., \& Rose, R. J. (2007b). Parental monitoring moderates the importance of genetic and environmental influences on adolescent smoking. Journal of Abnormal Psychology, 116, 213-218.

Duncan, L. E., \& Keller, M. C. (2011). A critical review of the first 10 years of candidate gene-by-environment interaction research in psychiatry. American Journal of Psychiatry, 168, 1041-1049.

Eaves, L. J. (2006). Genotype x environment interaction in psychopathology: Fact or artifact? Twin Research and Human Genetics, 9, 1-8.

Harden, K. P., Hill, J. E., Turkheimer, E., \& Emery, R. E. (2008). Gene-environment correlation and interaction in peer ef- fects on adolescent alcohol and tobacco use. Behavior Genetics, 38, 339-347.

Hicks, B. M., South, S. C., Dirago, A. C., Iacono, W. G., \& McGue, M. (2009). Environmental adversity and increasing genetic risk for externalizing disorders. Archives of General Psychiatry, 66, 640-648.

Kaprio, J., Pulkkinen, L., \& Rose, R. J. (2002). Genetic and environmental factors in health-related behaviors: Studies on Finnish twins and twin families. Twin Research, 5, 366371.

Kendler, K. S., \& Gardner, C. O. (2010). Interpretation of interactions: Guide for the perplexed. British Journal of Psychiatry, 197, 170-171.

Kendler, K. S., Gardner, C., \& Dick, D. M. (2011). Predicting alcohol consumption in adolescence from alcohol-specific and general externalizing genetic risk factors, key environmental exposures and their interaction. Psychological Medicine, 41, 1507-1516.

Lynch, M., \& Walsh, B. (1998). Genetics and analysis of quantitative traits. Sunderland, MA: Sinauer Associates.

Mather, K., \& Jinks, J. L. (1982). Biometrical genetics: The study of continuous variation. London: Chapman \& Hall.

McClelland, G. H., \& Judd, C. M. (1993). Statistical difficulties of detecting interactions and moderator effects. Psychological Bulletin, 114, 376-390.

Miles, D. R., Silberg, J. L., Pickens, R. W., \& Eaves, L. J. (2005). Familial influences on alcohol use in adolescent female twins: Testing for genetic and environmental interactions. Journal of Studies on Alcohol and Drugs, 66, 445-451.

Neale, M. C., Boker, S. M., Xie, G., \& Maes, H. (2003). Mx: Statistical modeling (6th ed.). Richmond VA: Department of Psychiatry.

Purcell, S. (2002). Variance components models for geneenvironment interaction in twin analysis. Twin Research, $5,554-571$.

Rathouz, P. J., Van Hulle, C. A., Rodgers, J. L., Waldman, I. D., \& Lahey, B. B. (2008). Specification, testing, and interpretation of gene-by-measured-environment interaction models in the presence of gene-environment correlation. Behavior Genetics, 38, 301-315.

Shanahan, M. J., \& Hofer, S. M. (2005). Social context in geneenvironment interactions: Retrospect and prospect. Journals of Gerontology Series B: Psychological Sciences and Social Science, 60, 65-76.

van der Sluis, S., Posthuma, D., \& Dolan, C. V. (2012). A note on false positives and power in $\mathrm{G} \times \mathrm{E}$ modelling of twin data. Behavior Genetics, 42, 170-186.

Veenstra, M. Y., Lemmens, P. H., Friesema, I. H., Garretsen, H. F., Knottnerus, J. A., \& Zwietering, P. J. (2006). A literature overview of the relationship between life-events and alcohol use in the general population. Alcohol and Alcoholism, 41, 455-463.

Verhulst, B., Neale, M. C., \& Kendler, K. S. (2015). The heritability of alcohol use disorders: A meta-analysis of twin and adoption studies. Psychological Medicine, 45, 10611072. 